\title{
Teaching technicians about animal research
}

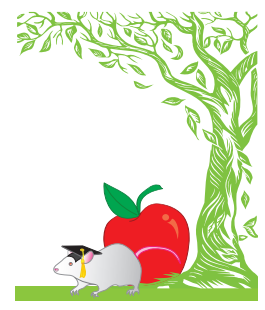

\author{
Chrystal L. Redding, MS, RLATg \& Stacey Oberhardt, BS, ALAT
}

A high rate of attrition among animal technicians in research can be costly for institutions, in part because of the cost and time needed to train new personnel. Technicians may leave their jobs for several reasons, including repetitive work environment, low pay, physically demanding work, unpleasant working conditions, emotional impact and, in many cases, minimal understanding of the research or why it is being done. Technicians generally receive training about the 'basic' policies and procedures in the first month of employment; these include the animal care and use program, alleviation of pain and distress, euthanasia methods, reporting of animal related concerns and species-specific care. This type of training is mandated in the Animal Welfare Act and Regulations, the Public Health Service Policy on Humane Care and Use of Laboratory Animals and the Guide for the Care and Use of Laboratory Animals. After the initial training, retraining occurs if there are problems and new training occurs when changes are made (e.g., a new species is planned for use or SOPs are being updated). But compliance with federal regulations shouldn't be the only motivation to train employees. Taking the time to train technicians in understanding the ethics and benefits of animal research may help to mitigate misunderstanding about the work done at the facility, which may in turn help to reduce attrition. In this way, providing more training can both increase employee satisfaction and decrease overall training costs.

In some cases, ethical concerns about animal research may be based on misunderstandings stemming from a lack of information. For this reason, institutions should make a commitment to inform staff members about the nature, objectives and benefits of the research done at the facilities where they work. Initially, such training

Redding and Oberhardt are members of the American Association for Laboratory Animal Science.

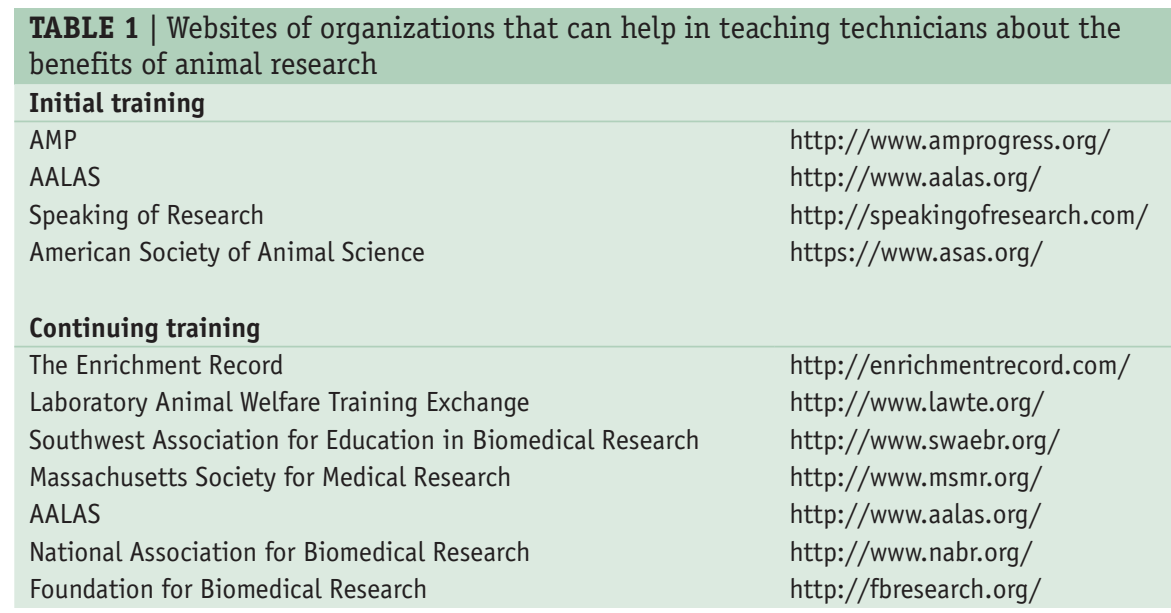

can be offered in a simple format such as a self-paced PowerPoint presentation or informational talk based on the talking points suggested by the organization Americans for Medical Progress (AMP; http://www. amprogress.org/amp_materials) or an informal 'town hall' meeting or interactive seminar given by a seasoned laboratory animal professional. AMP and the American Association for Laboratory Animal Science (AALAS) Foundation have produced videos called "Veterinarians Speaking for Research" (http://www.aalasfoundation.org/vfr. html) and "Careers in Laboratory Animal Science-Accept the Challenge to Care" (http://aalasfoundation.org/career_video. $\mathrm{html}$ ) that can be viewed online. These videos offer inexpensive training opportunities for technicians.

In addition to initial training, continuing education on the benefits of animal research will be informative to all involved and refresh key points. This can take the form of 'lunch and learn' sessions with principal investigators communicating about their research to a broad group of staff members. This interaction helps to build a relationship between investigators and technicians, who should feel engaged and better informed about the research. A technician with no research experience recently joined our facility; after basic regulatory training, she still did not understand the research component. She later spent a few hours with three different people involved in different aspects of the research: a principal investigator, a quality assurance investigator and a facility manager. Subsequently the technician stated that she had a clearer understanding of the importance of the research and her role in it. A number of organizations provide training materials and ideas or even speakers or webinars that can help in teaching technicians about the benefits of animal research (Table 1).

Initial and continuing education may help to lower attrition among technicians by enabling them to recognize the importance of their role in the research process. Education about the research purposes and ethical concerns associated with laboratory animals may lead technicians to remain in their positions longer, garnering valuable experience and knowledge. This increased knowledge base may create higher commitment to care, data collection and the science. In addition, establishing a comfortable understanding of research can facilitate transitions to AALAS certifications, to mentoring of more junior employees and to long careers in the laboratory animal science field. 